\title{
Pan Facial Fat Cells Transfer for Correction of Slim Face Deformity
}

\author{
DALIA M. RABEH, M.D.; IBRAHIM H. KAMEL, M.D. and AYMAN A. SHAKER, M.D. \\ The Department of Plastic, Burn and Maxillofacial Surgery, Faculty of Medicine, Ain Shams University, Cairo, Egypt
}

\begin{abstract}
Background: There are many reports in the literature describing how to slim down the face. These reports include diet and nutritional control, facial exercises, camouflage procedures by makeup and hair style and facial liposuction. However, excessive slimming of the face is due to decreased amount of subcutaneous fat cells in facial fat compartments.

Slim face deformity is the term which we applied to describe diffuse symmetrical hollowing of the face of young people without any apparent cause. It may be constitutional or due to massive weight loss in some patients. Fat grafting is one of many other techniques that were reported for correction and enhancement of localized facial regions and treatment of facial aging. However we could not find any report in the literature describing how to correct slim face deformity.
\end{abstract}

Obejective: The authors are going to describe slim face deformity and experience in correction using fat cells transfer in different facial fat compartments.

Patients and Methods: Thirty five patients with slim face deformity were treated by full face fat injection. The technique of fat harvesting, preparation and injection is illustrated. The patients were followed-up for at least six months.

Results: All patients had initial dissatisfaction with early post-operative appearance due to the surgical edema and overcorrection. They became gradually satisfied within a period of three months after surgery. $80 \%$ of patients had only one session of fat injection and only $20 \%$ of them asked for further fat injection and this was usually done eight to ten months after surgery.

Conclusion: Fat cell transfer, can be used as a safe long lasting procedure for correction of slim face deformity in patients who need full facial augmentation. It is a more natural and relatively cheaper technique in comparison to the use of other dermal fillers.

Keywords: Slim face - Facial contour deformity - Fat cell transfer - Facial fat compartments.

\section{INTRODUCTION}

Autologus whole fat graft transfer was introduced by Neuber in 1893. Although fat graft injection was practiced by Miller since 1926, it is only within the past 30 years that the popularity of autologus fat transfer for facial recontouring has increased within the Plastic Surgery Community. The transfer of autologus fat graft became a standardized technique for correction of facial contour deformities and aging [1].

There are many indications for facial fat grafting. The patients who can get benefit from this procedure can be classified into two groups. The first group includes patients of any age who have localized facial deformities due to any underlying pathology. In this group injection of fat is localized to certain areas according to the existing pathology [2]. The second group includes elder patients who have aging process related fat tissue redistribution. Since structural collapse is a hallmark of the aging face, structural fat grating for facial rejuvenation has been described extensively by Coleman [3]. A pan facial technique for structural autologous lipoaugmentation of the face was described by Donofrio [4]. This technique allows for even and complete volume replacement with minimal downtime and superior results. We noticed that there is a third group of patients who can also get benefit from this procedure. They are young patients having diffuse hollowing of the face without any underlying pathology and they seek for full face fat injection (pan facial fat cell transfer). We have given this configuration the term of slim face deformity. Slim face gives the patient the appearance of long face abnormality although they have no underlying bony framework abnormality. This deformity is constitutional but in some patients it became evident after massive weight loss. This loss may be the result of aggressive diet control or following bariatric surgery [5]. To our knowledge, slim face deformity was not previously described as a separate topic.

In this study we are going to describe the deformity and highlight the technique of fat cells transfer as a novel technique in management of this deformity. 


\section{PATIENTS AND METHODS}

After getting the approval from the Ethical Committee of Faculty of Medicine, Ain Shams University, this study included 35 patients. All patients were operated upon fat cells transfer for correction of slim face deformity during the period from January 2015 to December 2017.

All patients were young females ranging in age from 22 to 33 years. None of them requested the pan facial fat injection. In thirty patients the main request was cheek bone augmentation and malar enhancement by using fillers or Medpore Fig. (1). These patients were informed that malar (cheek bone) augmentation alone will not improve their aesthetic appearance and will get much benefit from pan facial augmentation.

Five patients were complaining from large nose and asking for getting smaller nose by reduction rhinoplasty Fig. (2). Those patients were informed that their noses are relatively huge due to associated facial hollowing and they will get better results if rhinoplasty is accompanied by full face augmentation.

Slim face deformity is charcterised by hollowing of temporal, glabellar and pre-auricular regions (the patient is looking older \& doleful), sharp contour of the face, depressed temporal fossae \& cheeks, depressed suborbital areas, sharp mandibular borders and there is no underlying pathology.

\section{Surgical technique:}

The patients were informed that they have to choose between two options, either to just inject the required amount of fat with the need for subsequent injection after few months or to overcorrect the deformity to obviate the need for a second secession. All of them accepted the second option.

Pre-operative mapping of the whole face is done and all the areas needed to be injected are marked. Photographs were taken before and after marking the skin and they were posted for intraoperative reference. The face is divided into three thirds, the upper, middle and lower thirds Fig. (3).

\section{Analysis of the slim face deformity:}

- Upper $1 / 3$ of the face: Glabellar region, central forehead. Temporal fossa.

- Middle 1/3 of the face: Pre-auricular region, cheek bone, upper lip.

- Lower 1/3 of the face: Mandibular border, chin, lower lip.
All cases were operated upon under general anesthesia with endotracheal nasal intubation using Ray's tube. The whole face has to be exposed with slight neck extension.

In the majority of patients, the abdomen was used as a donor site for the fat. In few patients who do not have abdominal fat or who want to do liposuction for other areas, the flanks or the thighs were used according to the patient's request. The chosen area was injected with modified Klein tumescent solution [6]. We used 500ml Ringer's solution containing $2 \mathrm{mg}$ epinephrine and $12 \mathrm{mEq}$ sodium bicarbonate [7]. Since all cases were done under G.A. no local anaesthetic was added to the tumescent solution. The injected amount depends on the aim of suction. If suction is needed only to get the fat required for injection, a maximum of $500 \mathrm{ml}$ will be sufficient.

The fat was aspirated using $4 \mathrm{~mm}$ blind cannula attached to $60 \mathrm{ml}$ syringe. The syringes filled with fat were left to stand with the plunger up for twenty minutes until the fat separates from the debris such blood, fibrin, and fluid. After removal of all fluid a further step of purification is done by allowing the remaining residual fat parcels and the fatty oil from the ruptured fat parcels to be decanted on a folded dry gauze absorb the fatty oil Fig. (4).

The first area to be injected is the temporal region [8] to correct temporal hollowness. The inlet for injection is in the front of the tragus. This is followed by injection of the contralateral temporal region, the injection is reaching to the hairline in a feathery way to avoid stepping at the hairline. Then the whole cheek [8] is injected starting with the mandibular border and going cephalolateral to the proper cheek area. Medially the injection has to reach to the nasolabial fold [8], caudally the injection has to extend below the mandibular border for further rounding of the face. Then the preauricular hollowness (lateral cheek region) is injected and this is repeated on the opposite side. Following this, the malar region is enhanced. The upper lip and or the lower lip are injected if there is indication for this. Lastly the glabellar depression is injected and if the central part of the forehead requires injection it followed [9].

The amount of fat required for injection as following:

Upper third of the face:

- Glabellar region: 3-5ml.

- Central forehead: 10-15ml.

- Temporal region: 10-20ml for each side. 


\section{Middle third of the face:}

- Preauricular region: 10-20ml for each side.

- Cheek bone: $10-15 \mathrm{ml}$ for each side.

- Upper lip: 3-5ml.

\section{Lower third of the face:}

- Mandibular border: 10-20ml for each side.

- Chin: 5-10ml.

- Lower lip: 3-5ml.
The total amount of injected fat ranged from 105 to $190 \mathrm{ml}$ in each patient according to the deformity. This amount included $30-50 \%$ excess for overcorrection.

The injected fat is immobilized in its position by applying of Elastoplast or face garment for the first three days. All patients were followed-up for at least six months after surgery. In few patients the maximum follow-up period was 3 years after surgery [10].
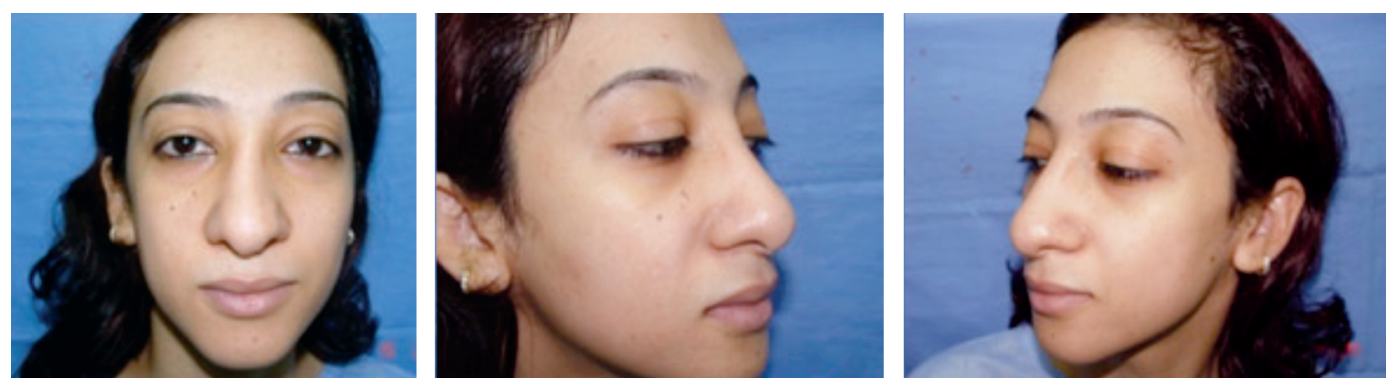

Fig. (1): 25 years old female was diagnosed as slim face deformity.
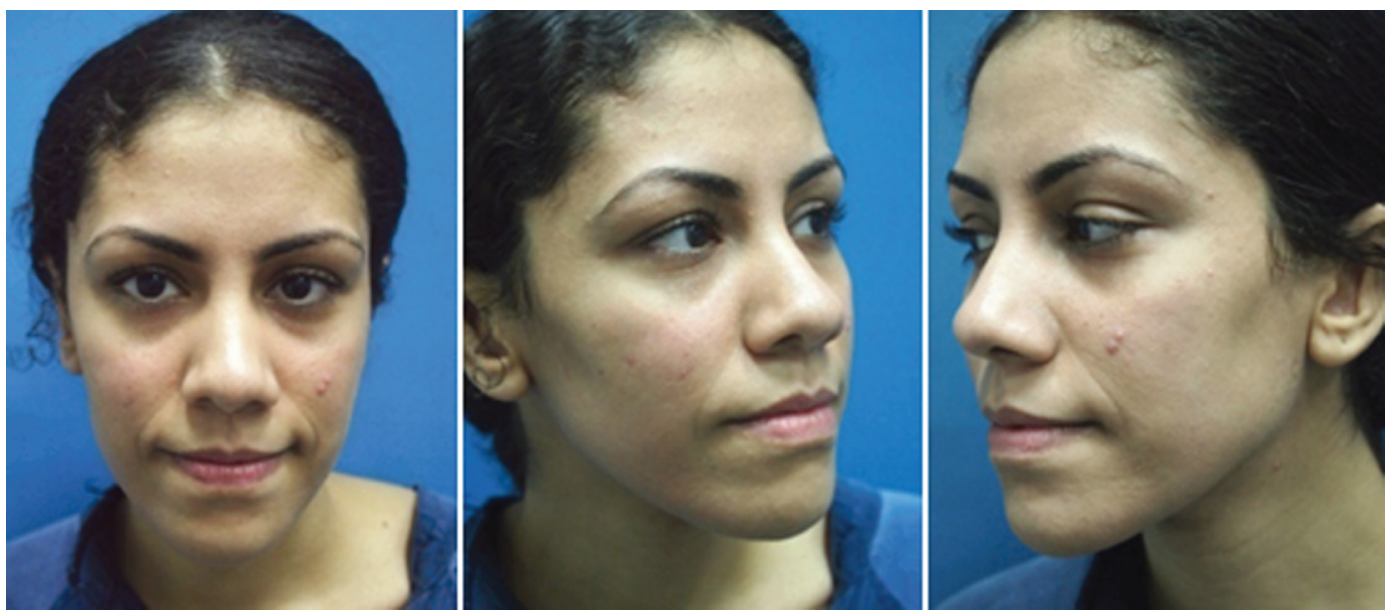

Fig. (2): 26 years old female was diagnosed as slim face deformity complaining of large nose.
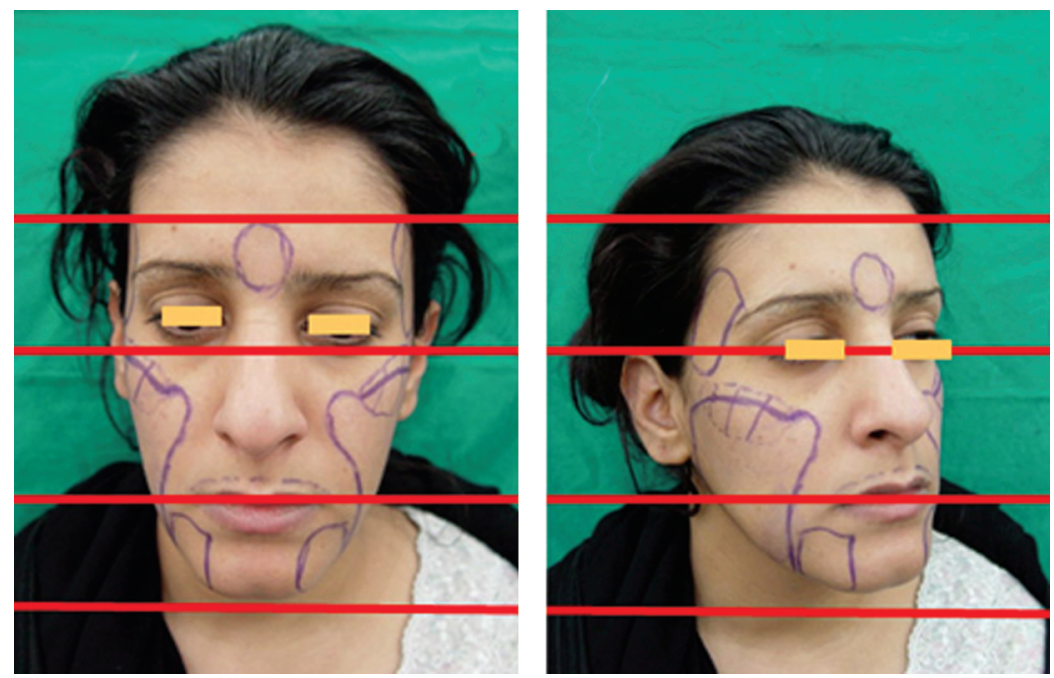

Fig. (3): Analysis of the slim face deformity. 

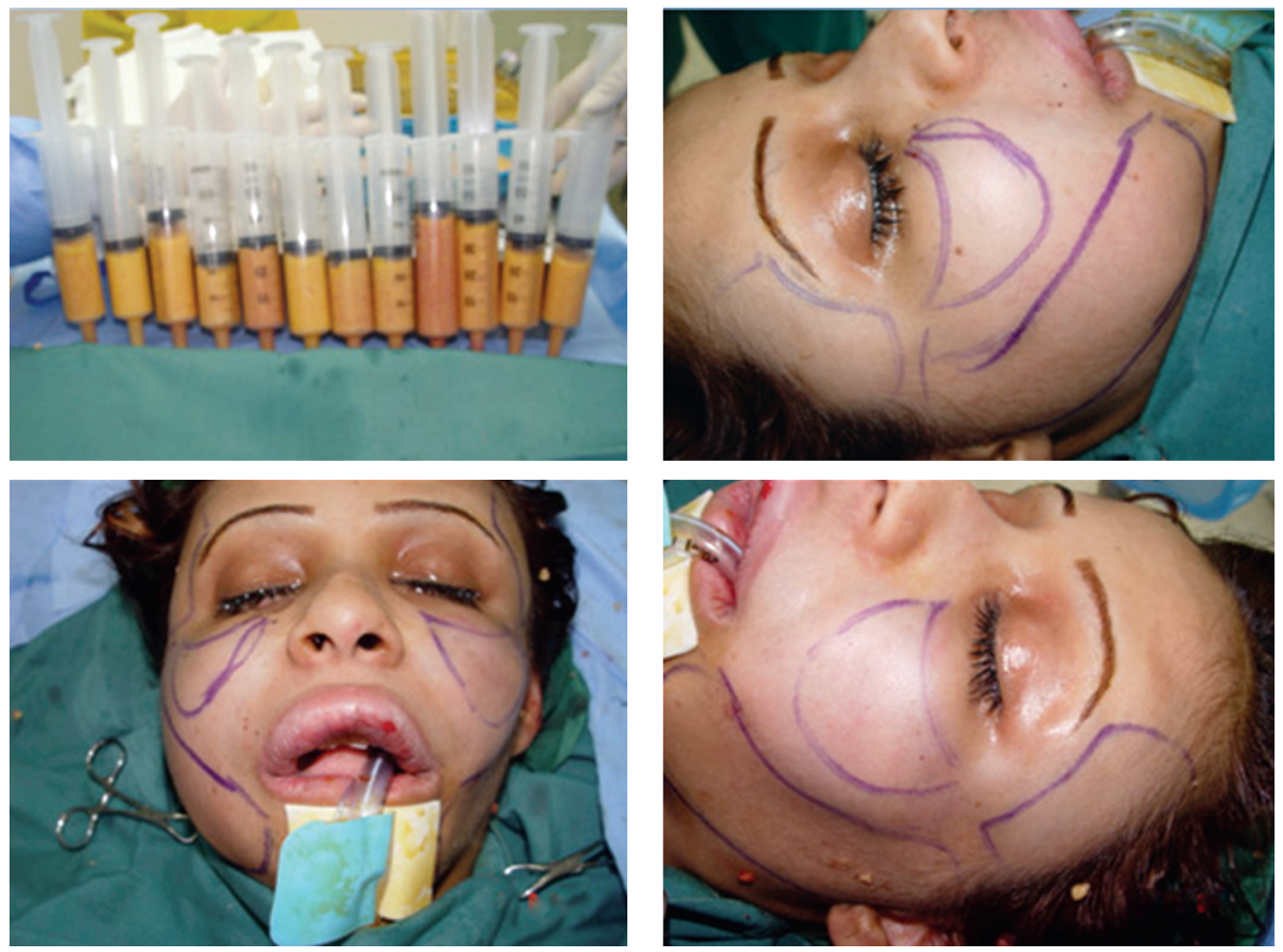

Fig. (4): Fat calls preparation and intra operative transfer to facial fat compartments in 24 years old female patient.

\section{RESULTS}

All patients showed initial dissatisfaction in the early post-operative period and they were worried about their looking Gradually with the subsidence of edema, the patients started to get satisfied with the results but they still have some concern about the appearance and the volume of their faces. Three to six months after surgery, most of the patients were completely satisfied with surgery Figs. (5-7). Eight to ten months after surgery about $20 \%$ of patients started to feel that they are going back to their original situation and they are asking about the possibility of further injection Fig. (8).

In only two patients after long follow-up period, the facial fat is increased in size. These patients were increased in body weight $[\mathbf{1 1 , 1 2 ] ~ F i g . ~ ( 7 ) . ~}$

Post-operative complications were minimal including edema and bruising which usually subside within one to two weeks after surgery Fig. (9). Minimal asymmetry between the two sides of the face were also noticed by some patients. No serious post-operative complications like fat embolism [13] were encountered in this series.
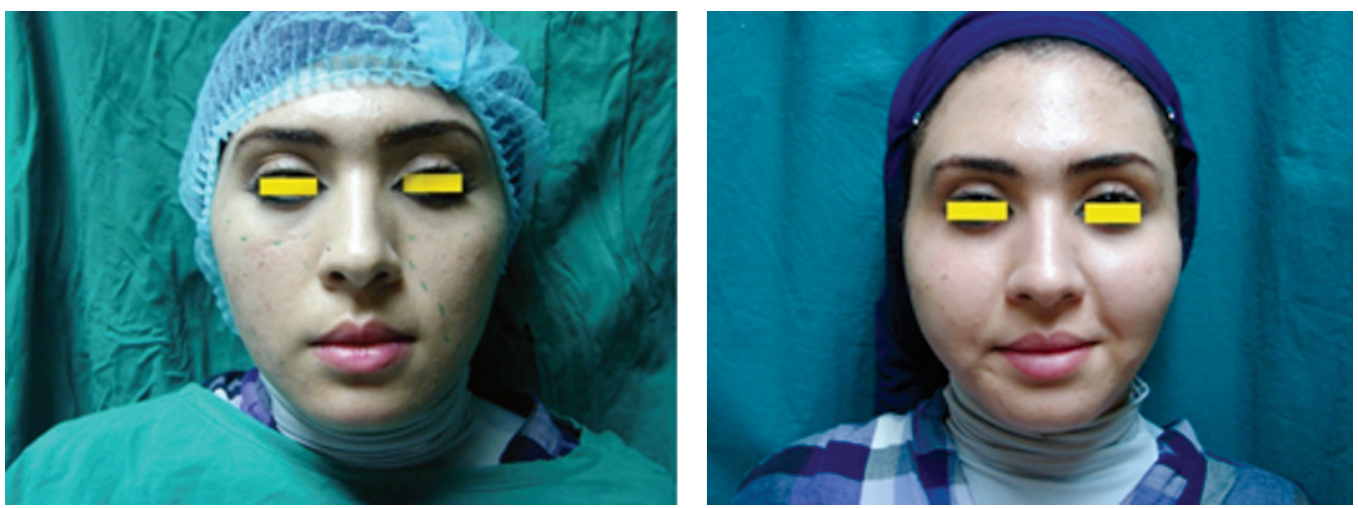

Fig. (5): 30 years old female patient, post-operative follow- up after 6 months. 
Fig. (6): 29 years old female patient, post-operative follow- up after 1 year.
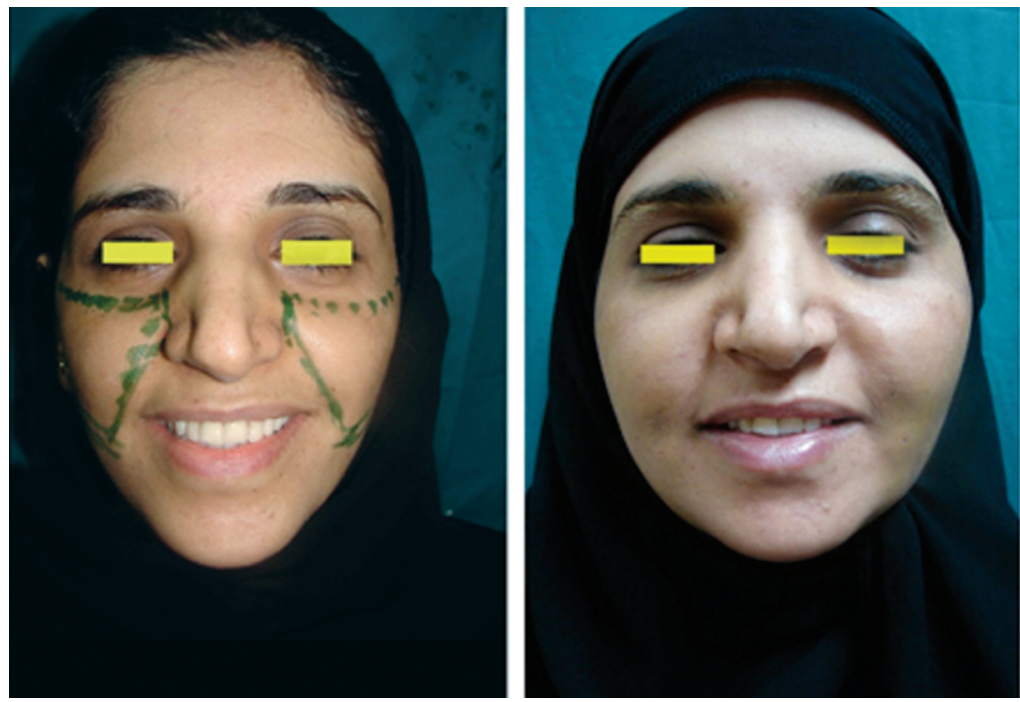

(A)
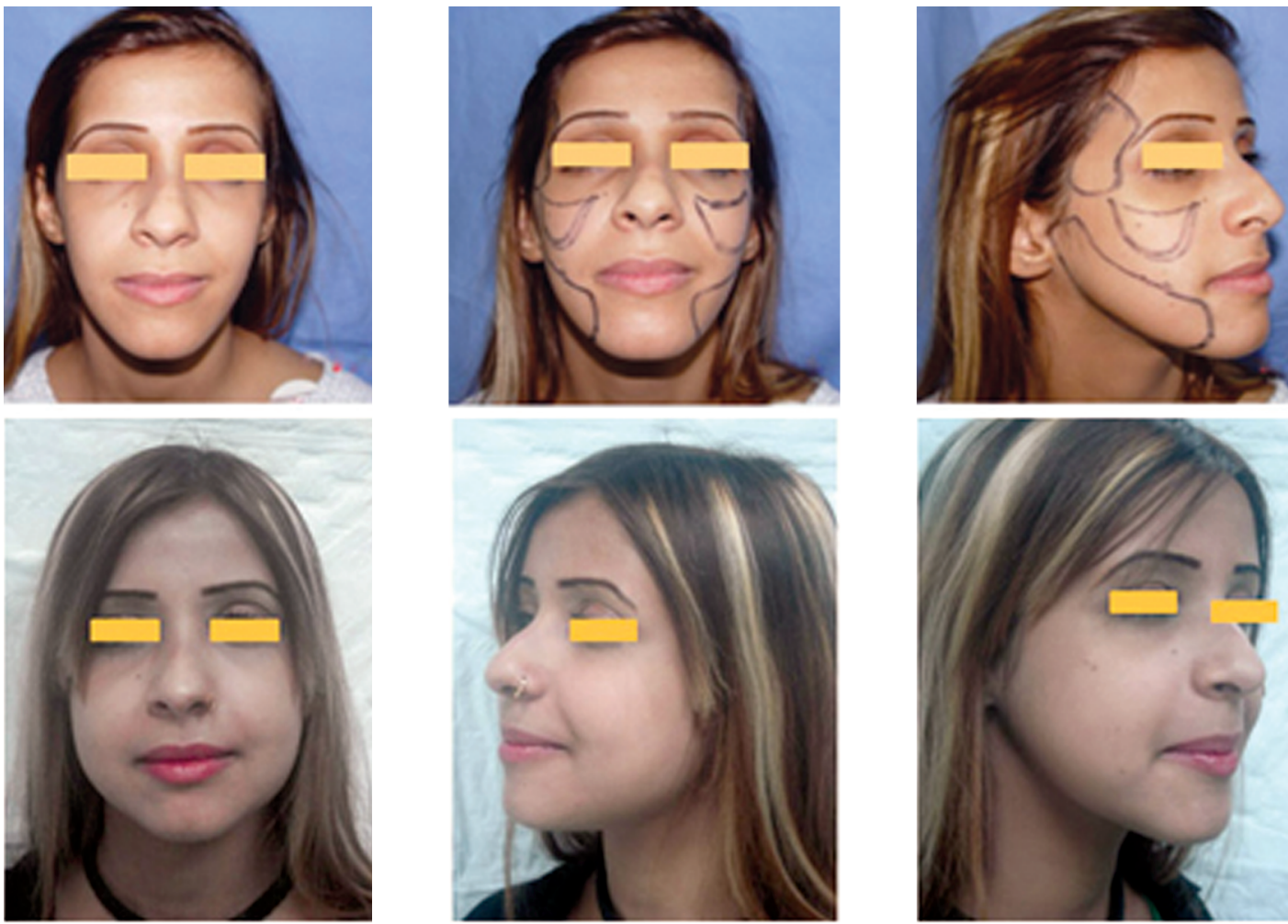

(B)
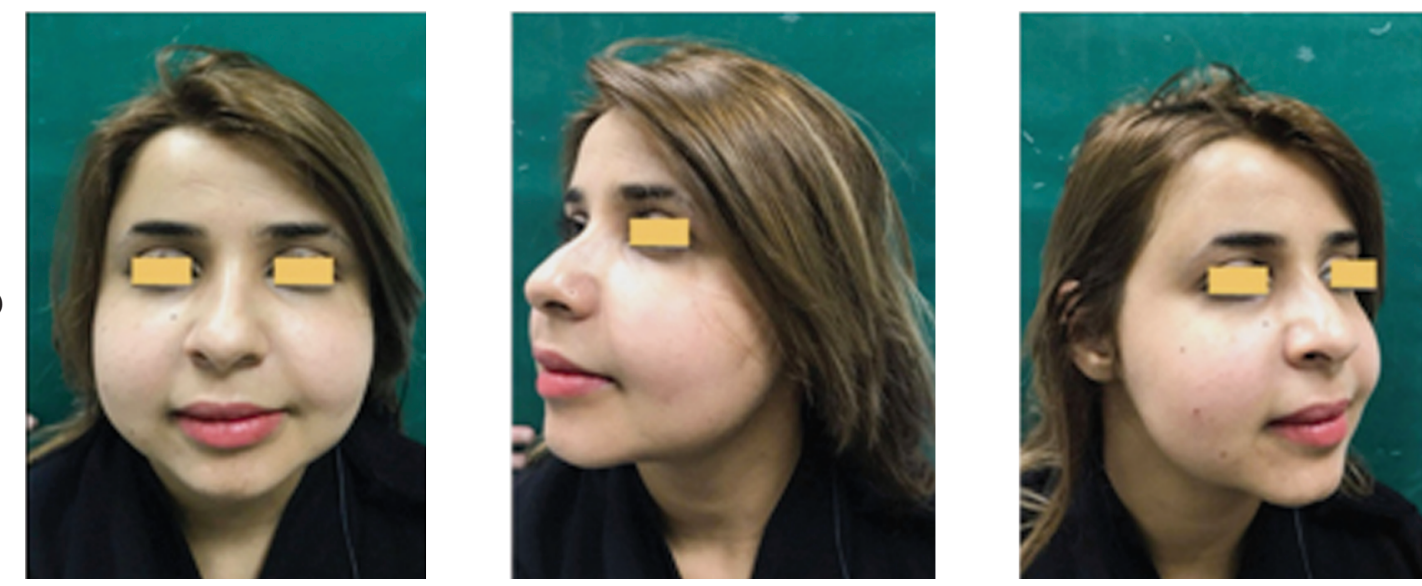

Fig. (7): 28 years old female patient (A) Pre-operative markings, (B) 3 months post-operative, (C) 1 year post- operative. 
Fig. (8): 29 years old female patient complaining of slim face deformity and large nose (A) Preoperative markings, (B) 10 months post-operative, she was not satisfied, marking for middle cheek and infra orbital regions re injection.
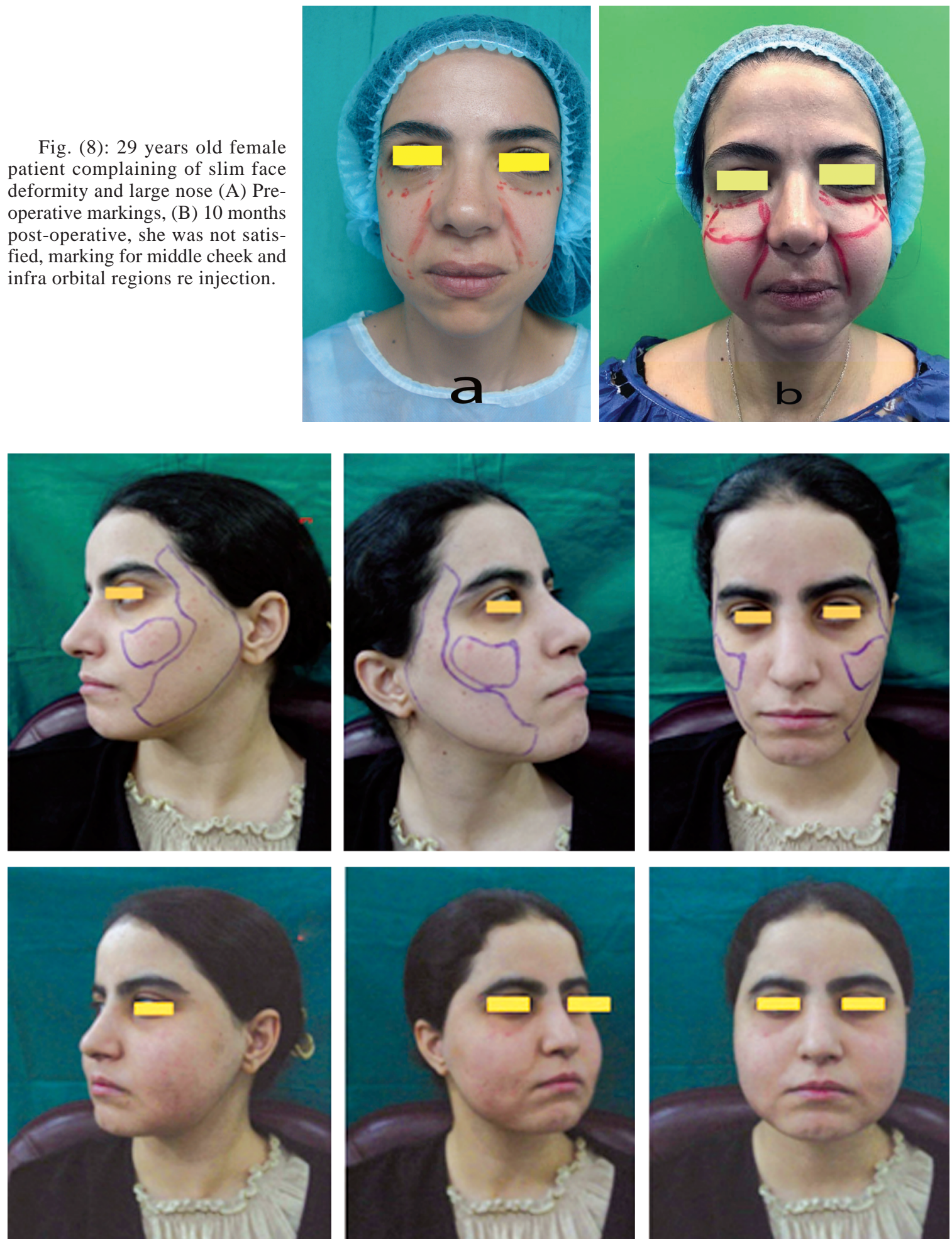

Fig. (9): 27 years old female patient, immediate post-operative show minimal edema and bruising.

\section{DISCUSSION}

Slim face deformity is the term that we have given to describe diffuse hollowing of the face without definite underlying pathology. It is a natural constitutional finding in some people. However, it may be presented or more aggravated after mas- sive weight loss. Many patients during the course of excessive diet regime complain that at certain point dieting causes slimming of their faces without any further reduction in the weight [14].

In this deformity the patient is looking older and doleful. The face has a sharp contour with 
depressed temporal fossa and hollowness of the lateral cheeks with sharp mandibular borders. Since there is no definite cause for the deformity, the term idiopathic lipodystrophy [15] can be used but the term, we describe, slim face is more descriptive.

The main deficient fat compartment in these patients are temporal, lateral cheek and mandibular. These fat compartments of the face were previously described by Rohrich et al., [8].

Though those patients have long and slender face they have to be differentiated from patients having long face syndrome. These patients have exaggerated anterior facial height due to maxillary vertical hyperplasia [16]. The differentiation can be done clinically and radiologically. In long face syndrome there is maxillary overjet and the cephalometric findingis conclusive for the diagnosis [16].

Another differential diagnosis is diffuse agerelated lipoatrophy (facial aging). It is obvious in our study that all patients are young ranging in age from 22-33 years without any manifestations of aging like wrinkles, furrows and associated skin manifestations of aging [17].

To address the problem of slim face, the deficient fat in facial fat compartments should be augmented with large amount of fat cell transfer. In this study the patient may be injected up to $190 \mathrm{ml}$ of fat and since the term of pan facial injection may be more descriptive. The advancement of recent techniques like combining the PRP with fat injection, the amount could be reduced with better and longer survival [18].

Our clinical mapping for the management of this deformity coincides with the previously reported articles by Rohrich et al., described the anatomical fat compartments. For example in the forehead region we divided it into 3 main compartments, the temporal region (fossa), the central forehead and glabellar region [8].

In most of the patients the abdomen was chosen as a donor site for fat harvesting because of its ease of access. This was also requested by patients to get rid from associated abdominal lipodystrophy [10]. To our knowledge there was no conclusive data confirming the superiority of one donor site over others. The only study that evaluated different donor sites was done by Rohrich et al., who concluded that no difference was found between four body sites including the abdomen, flank, thigh and medial knee [9].
Concerning the method of fat preparation, Rohrich et al., found no quantitative difference in adipose viability between non-centrifuged fat and fat that underwent the "cumbersome" technique of centrifugation [8]. This was also confirmed experimentally as they found no significance difference in fat graft weight and volume between fat prepared by centrifugation and fat prepared by sponge to wipe away fluids, oil, and debris. In addition, histologic studies revealed less fibrosis in the grafts that had not been centrifuged [18].

The only criticism for the technique is the question about the short-and long-term persistence of the transferred fat.

Guijarro-Martinez et al., showed progressive volumetric decrease for up to 6 months after the procedure [3]. Coleman believes graft volume stabilize at 3-4 months, although a subtle volumetric decrease may occur for up to one year; beyond that, it remains constant for 8-12 years. Because of the problem of resorption of the transplanted fat, 30-50\% over injection is ordinarily used [19].

Long-term results have been documented by surgeons practicing atraumatic harvesting and multilayer microdroplet infiltration, [19].

In two patients we noticed that over fat growth in long follow-up (one year) this may be due to increase body weight and the injected fat cells behave like the original fat cells in body [11,12].

Difficulties in assessing longevity are mainly due to lack of good objective measurement criteria. Photographs, though give an idea, yet they are two-dimensional representation of a threedimensional result. In our series long term followup results are more accurate than for those patients injected for facial rejuvenation. The reason is that aged patients usually start with augmentation and then often supplement their results with other rejuvenation procedures. The second reason is that they continue to age over the time as they are in follow-up. These two factors are not existing in our patients [18].

\section{Conclusion:}

Slim face is defined as a face who has diffuse hollowing of facial fat compartments without underlying pathology. Fat cell transfer, can be used as a safe long lasting procedure for correction of slim face deformity in patients who need pan facial augmentation. It is more natural and relatively cheaper technique in comparison to the use of other dermal fillers. 


\section{REFERENCES}

1- Ulrike Pilsl and Friedrich Anderhuber: The Chin and Adjacent Fat Compartments, Dermatol. Surg., 36: 2148, 2010 .

2- Matthew R. Kaufman, Timothy A. Miller, Catherine Huang, Jason Roostaien, Kristy L. Wasson, Rebekah K. Ashley and James P. Bradley: Autologous Fat Transfer for Facial Recontouring: Is There Science behind the Art? Plastic and Reconstructive Surgery, Volume 119, Number 7, 2012.

3- Raquel Guijarro-Martínez, Luis Miragall Alba, Mariano Marqués Mateo, Miguel Puche Torres and José Vicente Pascual Gil: Autologous fat transfer to the craniomaxillofacial region: Updates and controversies, Journal of Cranio-Maxillo-Facial Surgery xxx, 1-5, 2010.

4- Donofrio L.M.: Techniques in facial fat grafting. Aesthetic Surg. J., 28 (6): 681-7, 2008.

5- Guaraldi G., De Fazio D., Orlando G., Murri R., Wu A., Guaraldi P., et al.: Facial lipohypertrophyin HIV infected subjects who underwent autologousfat tissue transplantation. Clin. Infect. Dis., 40: e13, 2005.

6- Kaminer M.S. and Omura N.E.: Autologous fat transplantation. Arch. Dermatol., 137: 812, 2001.

7- Calabria R., et al.: Fat grafting: Fact or fiction? Aesthetic Surg., 25: 55, 2005.

8- Rod J. Rohrich, M.D. and Joel E. Pessa, M.D.: The Fat Compartments of the Face: Anatomy and Clinical Implications for Cosmetic Surgery, Plastic and Reconstructive Surgery. Volume 119, Number 7, 2012.

9- Rod J. Rohrich, M.D., Joel E. Pessa, M.D. and Brunno
Ristow, M.D.: The Youthful Cheek and the Deep Medial Fat Compartment, Plastic and Reconstructive Surgery, Volume 121, Number 6, 2012.

10- Guerrerosantos J.: Long-term outcome of autologous fat transplantation in aestheticfacial recontouring: Sixteen years of experience with 1936 cases. Clin. Plast. Surg., 27: 515e 543, 2000.

11- Miller J.J. and Popp J.C.: Fat hypertrophy after autologous fat transfer. Ophthal. Plast. Reconstr. Surg., 18: 228e231, 2002.

12- Latoni J.D., Marshall D.M. and Wolfe S.A.: Overgrowth of fat autotransplanted for correctionof localized steroidinduced atrophy. Plast. Reconstr. Surg., 106: 1566e1569, 2000 .

13- Yoon S.S., Chang D.I. and Chung K.C.: Acute fatal stroke immediately following autologous fat injection into the face. Neurology, 61: 1151e1152, 2003.

14- Fulton J.E., Suarez M., Silverton K. and Barnes T.: Small volumefat transfer. Dermatol. Surg., 24: 857-65, 1998.

15- Fulton J.E. Jr., Rahimi D.A., Helton P., Watson T. and Dahlberg K.: Lip rejuvenation. Dermatol. Surg., 26: 4745, 2000 .

16- Elsa Raskin and Gregory S. La Trenta: Why Do We Age in Our Cheeks? Aesthetic Surg. J., 27: 19-28, 2007.

17- Donofrio L.M.: Structural autologous lipoaugmentation: A pan- facial technique, Dermat Surg., 26: 1129-34, 2000.

18- Lisa M. Donofrio: Techniques in Facial Fat Grafting, Aesthetic Surgery Journal, 11, 12, 28: 681, 2011.

19- Coleman S.R.: Structural fat grafting: More than a permanent filler. Plast. Reconstr. Surg., 118: 108e120, 2006. 